\title{
Geared Robot Manipulators with a Jointed Unit: Kinematic Analysis and Its Application
}

\author{
Dar-Zen Chen,* Yu-Chu Huang \\ Department of Mechanical Engineering \\ National Taiwan University \\ Taipei, Taiwan, 10660 R.O.C. \\ Received 27 November 2000; accepted 15 March 2001
}

\begin{abstract}
An efficient and systematic methodology for the kinematic analysis of geared robot manipulators (GRMs) with a jointed unit is presented. It is shown that, by decomposing mechanical transmission lines of a GRM into serially connected jointed and disjointed units, kinematic relation between local inputs and local outputs of admissible jointed units can be systematically formulated. Accordingly, angular displacements of input links with respect to their associated primary links can be symbolically expressed in terms of joint angles by a unit-by-unit evaluation procedure. This unit-by-unit evaluation procedure provides better kinematic insights into how input torques are transmitted to various joints. It is also shown that an actuator in a GRM with a jointed unit can drive nonconsecutive joints by proper design of its gear train. A 3 degrees of freedom GRM with a jointed unit is used as an illustrative example. (C) 2001 John Wiley \& Sons, Inc.
\end{abstract}

\section{INTRODUCTION}

A robotic mechanism for industrial purpose is often in an open loop configuration to obtain a simple kinematic structure. However, the inertia loads caused by on-joint actuators inevitably degrade its dynamic performance. To reduce inertia loads, many robot manipulators are constructed in a partially closed-loop configuration. Under the partially

* To whom correspondence should be addressed; e-mail: dzchen@ccms.ntu.edu.tw. closed-loop concept, geared robot manipulators (GRMs) are widely adopted by using gear trains to transmit power from actuators which can be located as close to the ground as possible.

Several approaches for the topological synthesis of GRMs have been developed based on graph theory. ${ }^{1,2}$ Chang and Tsai $^{3}$ introduced the concept of mechanical transmission line (MTL) to describe the relation between joint torques and actuator torques of a GRM. Admissible structure matrices were generated to describe the coupling relations between MTLs. Chen and Shiue ${ }^{4}$ showed that an 
MTL can be decomposed into disjointed units including an input unit and several transmission units connected in series. Atlases of admissible input units and transmission units, which are classified as disjointed units, were enumerated. However, the graph representation of a GRM ${ }^{2}$ shown in Figure 1a cannot be obtained by applying the above methods. Chen et al. ${ }^{5}$ showed that MTLs may not necessarily be composed of disjointed units only and may also contain a 2 degree-of-freedom (DOF) nonfractionated geared kinematic chain, which is called a

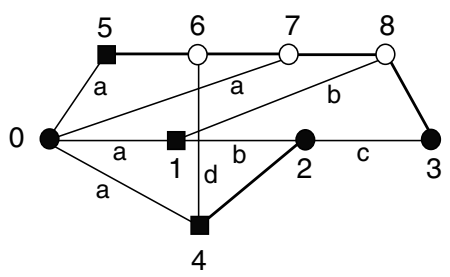

(a)

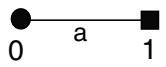

(b)

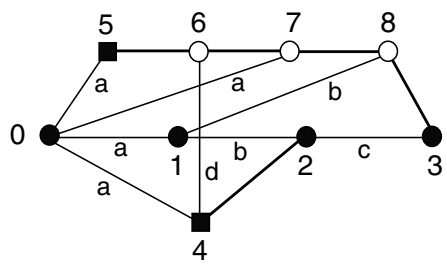

(c)

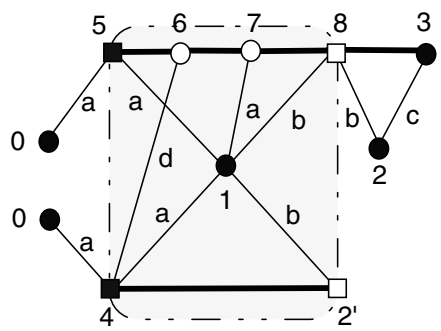

(d)

Figure 1. A 3-DOF GRM with a jointed unit. (a) The graph representation. (b) The disjointed MTL. (c) The jointed MTLs. (d) The pseudo-isomorphic subgraph of the jointed MTLs. jointed unit. With the atlases of admissible disjointed and jointed units, GRMs with a jointed unit with preferred DOF and number of links are enumerated. ${ }^{5}$

Various methods for the kinematic analysis of GRMs can be found in the literature. ${ }^{6-9}$ Among them, the most promising approach is perhaps the method of fundamental circuit and coaxial conditions introduced by Tsai. ${ }^{9}$ However, the analysis involves the manipulation of a set of linear equations simultaneously. Chen ${ }^{10}$ showed that a GRM can be decomposed into several MTLs and each MTL into 1 DOF disjointed unit connected in series. The input displacements can then be symbolically expressed in terms of the joint angles by a unit-byunit evaluation procedure. However, this unit-byunit evaluation procedure cannot be applied to the kinematic analysis of the GRM with a jointed unit as shown in Figure 1a.

In this article, the concept of jointed and disjointed MTLs will be applied to the kinematic analysis of a GRM with a jointed unit. It will be shown that, by decomposing a GRM with a jointed unit into jointed and disjointed MTLs and the jointed MTLs into jointed and disjointed units, input angular displacements of the jointed MTLs can be symbolically expressed in terms of joint angles by a unit-by-unit evaluation procedure. The advantage of this method is that algebraic expression for the kinematic relation between actuator space and joint space can be obtained without manipulating a set of linear equations simultaneously. In addition, this unit-by-unit evaluation procedure provides better kinematic insights into torque transmission among MTLs. It will also be shown that, in a GRM with a jointed unit, an actuator can drive nonconsecutive joints by properly choosing the jointed unit and disjointed units followed by gear train design.

\section{GEARED ROBOT MANIPULATORS}

In graph representation, links are denoted by vertices, turning pairs by thin edges, and gear pairs by heavy edges, and thin edges are labeled according to their axis locations in space. By rearranging coaxial turning pairs, the canonical graph representation ${ }^{9}$ can be obtained such that all edges lying on a thin-edged path traced from the base to any other vertex have different edge labels. Among these thin-edged paths, the linkage starting from the base 
and ending at the output link is defined as the equivalent open-loop chain. Links in the equivalent open-loop chain are referred to as primary links while all others are called secondary links. ${ }^{4}$ For the graph representation shown in Figure 1a, links 5, 1, and 4 are the input links and link 3 is the output link called the end-effector. Links $0,1,2$, and 3 are primary links and links $4,5,6,7$, and 8 are secondary links. In Figure 1a, primary links are denoted by solid nodes, input links by solid rectangles, and other links by hollow nodes.

Chang and $\mathrm{Tsai}^{3}$ showed that the kinematic structure of a GRM can be regarded as a combination of an equivalent open-loop chain and several MTLs. Each MTL represents a path to transmit the input motion to the end-effector. The arrangement of secondary links in an MTL determines where the actuators are located and how the input torque is transmitted to various joints. By collecting the vertices and edges associated with an MTL, an MTL subgraph can be identified.

Chen et al. ${ }^{5}$ showed that MTLs can be classified into disjointed MTL(s) and jointed MTLs. Figure 1b and $1 \mathrm{c}$ shows the direct-drive disjointed MTL and the jointed MTLs contained in Figure 1a, respectively. A disjointed MTL is composed of several 1 DOF disjointed units ${ }^{4}$ while a jointed MTL is composed of a 2-DOF jointed unit and several disjointed units. ${ }^{5}$ GRMs consisting of only disjointed MTLs are called disjointed GRMs while those with at least one set of jointed MTLs are called jointed GRMs.

Disjointed units can be further classified into input unit (IUs) and transmission units (TUs). Chen and Shiue ${ }^{4}$ enumerate admissible up to 5 link IUs and TUs as shown in Table Ia and b, respectively. In Table I, primary links are denoted by p, preconnecting links and postconnecting links are denoted, respectively, by $x$ and $y$, and secondary links are denoted by sequential numbers.

By separating common vertices on the equivalent open-loop chain and their associate joints followed by rearranging coaxial links, a pseudo-isomorphic subgraph of the jointed MTLs ${ }^{5}$ in Figure 1c can be obtained as shown in Figure 1d. In Figure 1d, links 5 and 4 are the preconnecting links while links 8 and $2^{\prime}$ are the postconnecting links of the jointed unit enclosed by a dashed rectangle. Postconnecting links 8 and $2^{\prime}$ are coaxial with primary links 1 and 2 while preconnecting links 5 and 4 are coaxial with primary links 0 and 1 . These coaxial conditions lead to the formation of articulated joints on the equivalent open-loop chain. Table II shows the admissible
Table I. Admissible disjointed units and their forward gains: a) input units; b) transmission units.

\begin{tabular}{|c|c|c|c|}
\hline & $g_{I U}$ & $g_{I U}$ \\
\hline & & & \\
\hline \\
\hline
\end{tabular}

(a)

\begin{tabular}{|c|c|c|c|}
\hline & gTU & & gTU \\
\hline 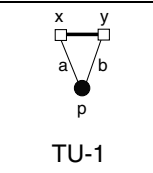 & $e_{y x}$ & 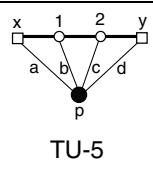 & $e_{y 2} e_{21} e_{1 x}$ \\
\hline $\mathrm{TU}_{\mathrm{p}}^{\mathrm{x}}$ & $e_{y 1} e_{1 x}$ & $\int_{\mathrm{p}}^{\mathrm{TU}-6^{*}} \mathrm{a}^{\mathrm{x}} \mathrm{b}$ & $\frac{e_{y 2}}{1 e_{p 1} e_{12}}$ \\
\hline 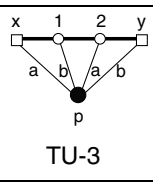 & $e_{y 2} e_{21} e_{1 x}$ & TU-7 ${ }^{*}$ & $\frac{e_{1 x}}{1 e_{p 2} e_{2 y}}$ \\
\hline${ }_{T U-4^{*}}^{x}$ & $e_{y 2} e_{21} e_{1 x}$ & & \\
\hline
\end{tabular}

(b)

jointed units enumerated from 2 DOF nonfractionated $\mathrm{GKCs}^{11}$ by Chen et al. $^{5}$ In Table II, primary links are denoted by $\mathrm{p}$, upper and lower local inputs are denoted, respectively, by $x_{\mathrm{U}}$ and $x_{\mathrm{L}}$, upper and lower local outputs are denoted, respectively, by $y_{U}$ and $y_{\mathrm{L}}$ and secondary links are denoted by sequential numbers. 
Table II. Admissible jointed units and their forward gain matrices.

\begin{tabular}{|c|c|c|}
\hline & $\mathbf{G}_{J}$ & $\mathbf{G}_{\mathrm{J}, 11}+\mathbf{G}_{\mathrm{J}, 12}$ \\
\hline $\mathrm{JU}_{\mathrm{J}-1}^{\mathrm{y}_{\mathrm{L}}}$ & {$\left[\begin{array}{cc}e_{1 x_{U}} e_{21} e_{y_{U} 2} & e_{y_{L} x_{L}}\left(1-e_{1 x_{U}} e_{21}\right) \\
0 & e_{y_{L} x_{L}}\end{array}\right]$} & $\begin{array}{c}e_{1 x_{U}} e_{21} e_{y_{U} 2} \\
+e_{y_{L} x_{L}}\left(1-e_{1 x_{U}} e_{21}\right)\end{array}$ \\
\hline 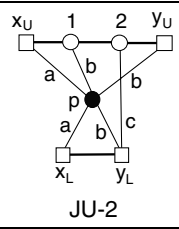 & {$\left[\begin{array}{cc}e_{1 x_{U}} e_{21} e_{y_{U} 2} & e_{1 x_{U}}\left(1-e_{21} e_{y_{U} 2}\right) \\
0 & e_{y_{L} x_{L}}\end{array}\right]$} & $e_{1 x_{u}}$ \\
\hline 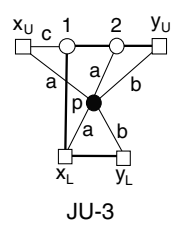 & {$\left[\begin{array}{cc}\frac{e_{y_{U} 2}}{1-e_{12} e_{x_{L} 1}} & \frac{e_{x_{L} 1} e_{12} e_{y_{L} x_{L}}}{e_{12} e_{x_{L} 1}-1} \\
0 & e_{y_{L} x_{L}}\end{array}\right.$} & $\frac{e_{x_{L} 1} e_{12} e_{y_{L} x_{L}}-e_{y_{U} 2}}{e_{12} e_{x_{L} 1}-1}$ \\
\hline $\mathrm{JU}_{\mathrm{J}-4}^{\mathrm{x}_{\mathrm{L}}}$ & {$\left[\begin{array}{cc}e_{1 x_{U}}\left(1-e_{21} e_{y_{L} 2}\right) & e_{1 x_{U}} e_{21} e_{y_{L} 2} \\
0 & e_{y_{L} x_{L}}\end{array}\right]$} & $e_{1 x_{u}}$ \\
\hline (n) & {$\left[\begin{array}{cc}\frac{e_{1 x_{U}}}{1-e_{y_{L} 2} e_{2 y_{U}}} & \frac{e_{1 x_{U}} e_{y_{L} 2} e_{2 y_{U}}}{e_{y_{L} 2} e_{2 y_{U}}-1} \\
0 & e_{y_{L} x_{L}}\end{array}\right]$} & $e_{1 x_{u}}$ \\
\hline
\end{tabular}

\section{SPEED RATIO OF DISJOINTED AND JOINTED UNITS}

Let links $j$ and $k$ be a gear pair and let link $i$ be its carrier. The associated fundamental circuit equation $^{12}$ for fundamental circuit $(j, k)(i)$ can be written as

$$
q_{j, i}=e_{k j} q_{k, i}
$$

where $q_{j, i}$ denotes relative angular displacement of link $j$ with respect to link $i, e_{k j}= \pm N_{k} / N_{j}$ represents the gear ratio of the gear pair mounted on links $k$ and $j$, positive or negative accordingly as a positive rotation of link $k$ results in a positive or negative rotation of link $j$ about their predefined axes of rotation, and $N_{k}$ is the number of teeth on link $k$.
Chen $^{10}$ showed that angular displacements of local input and output with respect to the primary link of a disjointed unit can be related by its forward gain. Table Ia and $b$ shows the forward gains of admissible IUs and TUs, respectively. Taking IU-2 in Table Ia as an example, we have

$$
q_{x, p}=g_{\mathrm{IU}-2} q_{y, p}=e_{y 1} e_{1 x} q_{y, p}
$$

where $g_{\text {IU-2 }}$ is the forward gain of IU-2.

For jointed unit JU-1 in Table II, fundamental circuit equations associated with fundamental circuits $\left(x_{\mathrm{U}}, 1\right)\left(x_{\mathrm{L}}\right),(1,2)\left(x_{\mathrm{L}}\right),\left(2, y_{\mathrm{U}}\right)(\mathrm{p})$ and $\left(x_{\mathrm{L}}\right.$, $\left.y_{\mathrm{L}}\right)(\mathrm{p})$ can be written as

$$
q_{x_{\mathrm{U}}, x_{\mathrm{L}}}=e_{1 x_{\mathrm{U}}} q_{1, x_{\mathrm{L}}}
$$




$$
\begin{gathered}
q_{1, x_{\mathrm{L}}}=e_{21} q_{2, x_{\mathrm{L}}} \\
q_{2, \mathrm{p}}=e_{y_{\mathrm{U}} 2} q_{y_{\mathrm{U}}, \mathrm{p}}
\end{gathered}
$$

and

$$
q_{x_{\mathrm{L}}, \mathrm{p}}=e_{y_{\mathrm{L}} x_{\mathrm{L}}} q_{y_{\mathrm{L}}, \mathrm{p}}
$$

The relation among relative angular displacements of the two sets of coaxial links, $\left(x_{\mathrm{U}}, \mathrm{p}, x_{\mathrm{L}}\right)$ and $\left(2, \mathrm{p}, x_{\mathrm{L}}\right)$, can be obtained as

$$
q_{x_{\mathrm{U}}, x_{\mathrm{L}}}=q_{x_{\mathrm{U}}, \mathrm{p}}+q_{\mathrm{p}, x_{\mathrm{L}}}
$$

and

$$
q_{2, x_{\mathrm{L}}}=q_{2, \mathrm{p}}+q_{\mathrm{p}, x_{\mathrm{L}}}
$$

By solving Eqs. (3)-(8), angular displacements of local inputs and local outputs with respect to the primary link of jointed unit JU-1 can be related as

$$
\left[\begin{array}{c}
q_{x_{\mathrm{U}}, \mathrm{p}} \\
q_{x_{\mathrm{L}}, \mathrm{p}}
\end{array}\right]=\left[\begin{array}{cc}
e_{1 x_{\mathrm{U}}} e_{21} e_{y_{\mathrm{U}} 2} & e_{y_{\mathrm{L}} x_{\mathrm{L}}}\left(1-e_{1 x_{\mathrm{U}}} e_{21}\right) \\
0 & e_{y_{\mathrm{L}} x_{\mathrm{L}}}
\end{array}\right]\left[\begin{array}{c}
q_{y_{\mathrm{U}}, \mathrm{p}} \\
q_{y_{\mathrm{L}}, \mathrm{p}}
\end{array}\right]
$$

or

$$
\boldsymbol{\Theta}_{x, \mathrm{p}}=\mathbf{G}_{\mathrm{JU}-1} \boldsymbol{\Theta}_{y, \mathrm{p}}
$$

where $\boldsymbol{\Theta}_{x, \mathrm{p}}=\left[q_{x_{\mathrm{U}}, \mathrm{p}}, q_{x_{\mathrm{L}} \mathrm{p}}\right]^{T}, \quad \boldsymbol{\Theta}_{y, \mathrm{p}}=\left[q_{y_{\mathrm{U}}, \mathrm{p}}, q_{y_{\mathrm{L}}, \mathrm{p}}\right]^{T}$, and $\mathbf{G}_{\mathrm{JU}-1}$ is the forward gain matrix associated with JU-1.

Table II shows the forward gain matrices of admissible jointed units. Note that the $(2,1)$ element of each forward gain matrix is always zero. Hence, the relation between local inputs and local outputs for a jointed unit J can be generally expressed as

$$
\left[\begin{array}{l}
q_{x_{\mathrm{U}}, \mathrm{p}} \\
q_{x_{\mathrm{L}}, \mathrm{p}}
\end{array}\right]=\left[\begin{array}{cc}
\mathbf{G}_{\mathrm{J}, 11} & \mathbf{G}_{\mathrm{J}, 12} \\
0 & \mathbf{G}_{\mathrm{J}, 22}
\end{array}\right]\left[\begin{array}{l}
q_{y_{\mathrm{U}}, \mathrm{p}} \\
q_{y_{\mathrm{L}}, \mathrm{p}}
\end{array}\right]
$$

\section{KINEMATIC PROPAGATION OF UNITS}

Figure 2 shows a set of typical jointed MTLs with a jointed unit J and several optionally connected disjointed units. The jointed MTLs are separated along the equivalent open-loop chain into an upper subMTL and a lower sub-MTL with jointed unit J in common. The preconnecting links $x_{U}$ and $x_{\mathrm{L}}$ of jointed unit J are used as local input links connecting to preceding disjointed unit(s) or can be used as

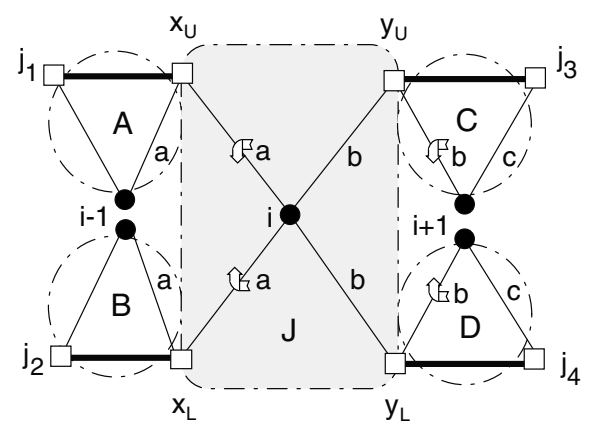

Figure 2. A set of typical jointed MTLs.

input links of the jointed MTLs. The postconnecting links $y_{\mathrm{U}}$ and $y_{\mathrm{L}}$ of jointed unit $\mathrm{J}$ are used as local output links connecting to succeeding disjointed unit(s). Kinematic analysis of the jointed MTLs can be divided into the following cases.

\subsection{Relocation of Inputs}

The function of a preceding disjointed unit to a joint unit can be regarded as the relocation of input link. For the case that preceding disjointed unit $\mathrm{A}$ is connected to jointed unit J, as shown in Figure 2, the input link is relocated from link $x_{\mathrm{U}}$ to link $j_{1}$. From Eq. (2), angular displacements of link $j_{1}$ and link $x_{\mathrm{U}}$ with respect to primary link $i-1$ of disjointed unit A are related as

$$
q_{j_{1}, i-1}=g_{\mathrm{A}} q_{x_{\mathrm{U}}, i-1}
$$

Since links $i-1, x_{\mathrm{U}}$, and $i$ are coaxial, we have

$$
q_{x_{\mathrm{U}}, i-1}=q_{x_{\mathrm{U}}, i}+q_{i, i-1}=q_{x_{\mathrm{U}}, i}+\theta_{i}
$$

where the angular displacement of primary link $i$ with respect to link $i-1, q_{i, i-1}$, is denoted by the joint angular displacement $\theta_{i}$.

Substituting Eq. (13) into Eq. (12) yields

$$
q_{j_{1}, i-1}=g_{\mathrm{A}} \theta_{i}+g_{\mathrm{A}} q_{x_{\mathrm{U}}, i}
$$

From Eq. (14), angular displacements of links $j_{1}$ and $x_{\mathrm{L}}$ with respect to their associated primary links can be written as

$$
\left[\begin{array}{c}
q_{j_{1}, i-1} \\
q_{x_{\mathrm{L}}, i}
\end{array}\right]=\left[\begin{array}{c}
g_{\mathrm{A}} \\
0
\end{array}\right] \theta_{i}+\left[\begin{array}{cc}
g_{\mathrm{A}} & 0 \\
0 & 1
\end{array}\right]\left[\begin{array}{l}
q_{x_{\mathrm{U}}, i} \\
q_{x_{\mathrm{L}}, i}
\end{array}\right]
$$


Similarly, for the case that preceding disjointed unit $\mathrm{B}$ is connected to jointed unit $\mathrm{J}$ to relocate its local input link from $x_{\mathrm{L}}$ to link $j_{2}$, angular displacements of links $j_{2}$ and $x_{U}$ with respect to their associated primary links can be related as

$$
\left[\begin{array}{c}
q_{x_{\mathrm{U}}, i} \\
q_{j_{2}, i-1}
\end{array}\right]=\left[\begin{array}{c}
0 \\
g_{\mathrm{B}}
\end{array}\right] \theta_{i}+\left[\begin{array}{cc}
1 & 0 \\
0 & g_{\mathrm{B}}
\end{array}\right]\left[\begin{array}{c}
q_{x_{\mathrm{U}}, i} \\
q_{x_{\mathrm{L}}, i}
\end{array}\right]
$$

Hence, for a jointed unit $\mathrm{J}$ with two preceding disjointed units $\mathrm{A}$ and $\mathrm{B}$, angular displacements of input links, $j_{1}$ and $j_{2}$, with respect to their associated primary links can be related as

$$
\left[\begin{array}{l}
q_{j_{1}, i-1} \\
q_{j_{2}, i-1}
\end{array}\right]=\left[\begin{array}{l}
g_{\mathrm{A}} \\
g_{\mathrm{B}}
\end{array}\right] \theta_{i}+\left[\begin{array}{cc}
g_{\mathrm{A}} & 0 \\
0 & g_{\mathrm{B}}
\end{array}\right]\left[\begin{array}{l}
q_{x_{\mathrm{U}}, i} \\
q_{x_{\mathrm{L}}, i}
\end{array}\right]
$$

\subsection{Extension of the Jointed Unit}

The connection of disjointed units succeeding a jointed unit in its upper and/or lower postconnecting links can be viewed as the extension of the jointed unit. For the case that succeeding disjointed unit $\mathrm{C}$ is connected to jointed unit $\mathrm{J}$ to extend its local output link from $y_{U}$ to link $j_{3}$, angular displacements of link $y_{U}$ and link $j_{3}$ with respect to primary link $i+1$ of the disjointed unit $C$ can be related according to Eq. (2) as

$$
q_{y_{\mathrm{U}}, i+1}=g_{\mathrm{C}} q_{j_{3}, i+1}
$$

Since links $i, y_{\mathrm{U}}$, and $i+1$ are coaxial, the coaxial equation can be written as

$$
q_{y_{U}, i}=q_{y_{U}, i+1}+q_{i+1, i}=q_{y_{U}, i+1}+\theta_{i+1}
$$

Substituting Eq. (18) into Eqs. (19) yields

$$
q_{y_{\mathrm{U}}, i}=g_{\mathrm{C}} q_{j_{3}, i+1}+\theta_{i+1}
$$

From Eq. (20), angular displacements of links $y_{U}$ and $y_{L}$ with respect to their associated primary links can be written as

$$
\left[\begin{array}{l}
q_{y_{\mathrm{U}}, i} \\
q_{y_{\mathrm{L}}, i}
\end{array}\right]=\left[\begin{array}{l}
1 \\
0
\end{array}\right] \theta_{i+1}+\left[\begin{array}{cc}
g_{\mathrm{C}} & 0 \\
0 & 1
\end{array}\right]\left[\begin{array}{c}
q_{j_{3}, i+1} \\
q_{y_{\mathrm{L}}, i}
\end{array}\right]
$$

Similarly, for the case that succeeding disjointed unit $\mathrm{D}$ is connected to jointed unit $\mathrm{J}$ to extend its local output link from $y_{\mathrm{L}}$ to link $j_{4}$, angular displacements of links $y_{\mathrm{U}}$ and $y_{\mathrm{L}}$ with respect to their associated primary links can be written as

$$
\left[\begin{array}{l}
q_{y_{\mathrm{U}}, i} \\
q_{y_{\mathrm{L}}, i}
\end{array}\right]=\left[\begin{array}{l}
0 \\
1
\end{array}\right] \theta_{i+1}+\left[\begin{array}{cc}
1 & 0 \\
0 & g_{\mathrm{D}}
\end{array}\right]\left[\begin{array}{c}
q_{y_{\mathrm{U}}, i} \\
q_{j_{4}, i+1}
\end{array}\right]
$$

Hence, for a jointed unit J with two succeeding disjointed units $C$ and $D$, angular displacements of input links, $y_{U}$ and $y_{L}$, with respect to their associated primary links can be related as

$$
\left[\begin{array}{l}
q_{y_{\mathrm{U}}, i} \\
q_{y_{\mathrm{L}}, i}
\end{array}\right]=\left[\begin{array}{l}
1 \\
1
\end{array}\right] \theta_{i+1}+\left[\begin{array}{cc}
g_{\mathrm{C}} & 0 \\
0 & g_{\mathrm{D}}
\end{array}\right]\left[\begin{array}{l}
q_{j_{3}, i+1} \\
q_{j_{4}, i+1}
\end{array}\right]
$$

\subsection{Merged Result}

For the case that postconnecting links $j_{3}$ and $j_{4}$ are combined as a primary link of the equivalent openloop chain, link $i+2$, Eq. (23) can be written as

$$
\begin{aligned}
{\left[\begin{array}{l}
q_{y_{\mathrm{U}}, i} \\
q_{y_{\mathrm{L}}, i}
\end{array}\right] } & =\left[\begin{array}{l}
1 \\
1
\end{array}\right] \theta_{i+1}+\left[\begin{array}{cc}
g_{\mathrm{C}} & 0 \\
0 & g_{\mathrm{D}}
\end{array}\right]\left[\begin{array}{l}
q_{i+2, i+1} \\
q_{i+2, i+1}
\end{array}\right] \\
& =\left[\begin{array}{ll}
1 & g_{\mathrm{C}} \\
1 & g_{\mathrm{D}}
\end{array}\right]\left[\begin{array}{l}
\theta_{i+1} \\
\theta_{i+2}
\end{array}\right]
\end{aligned}
$$

By substituting Eq. (24) into Eq. (11), angular displacements of local inputs of a jointed unit J with respect to its primary link $i$ can then be related as

$$
\begin{aligned}
{\left[\begin{array}{l}
q_{x_{\mathrm{U}}, i} \\
q_{x_{\mathrm{L}}, i}
\end{array}\right]=} & {\left[\begin{array}{cc}
\mathbf{G}_{\mathrm{J}, 11} & \mathbf{G}_{\mathrm{J}, 12} \\
0 & \mathbf{G}_{\mathrm{J}, 22}
\end{array}\right]\left[\begin{array}{ll}
1 & g_{\mathrm{C}} \\
1 & g_{\mathrm{D}}
\end{array}\right]\left[\begin{array}{c}
\theta_{i+1} \\
\theta_{i+2}
\end{array}\right] } \\
= & {\left[\begin{array}{cc}
\mathbf{G}_{\mathrm{J}, 11}+\mathbf{G}_{\mathrm{J}, 12} & \mathbf{G}_{\mathrm{J}, 11} \cdot g_{\mathrm{C}}+\mathbf{G}_{\mathrm{J}, 12} \cdot g_{\mathrm{D}} \\
\mathbf{G}_{\mathrm{J}, 22} & \mathbf{G}_{\mathrm{J}, 22} \cdot g_{\mathrm{D}}
\end{array}\right] } \\
& \times\left[\begin{array}{c}
\theta_{i+1} \\
\theta_{i+2}
\end{array}\right]
\end{aligned}
$$

By substituting Eq. (25) into Eq. (17), angular displacements of input links, $j_{1}$ and $j_{2}$, with respect to their associated primary links are related to joint displacements of the jointed MTLs as

$$
\begin{aligned}
{\left[\begin{array}{l}
q_{j_{1}, i-1} \\
q_{j_{2}, i-1}
\end{array}\right]=} & {\left[\begin{array}{l}
g_{\mathrm{A}} \\
g_{\mathrm{B}}
\end{array}\right] \theta_{i}+\left[\begin{array}{cc}
g_{\mathrm{A}} & 0 \\
0 & g_{\mathrm{B}}
\end{array}\right] } \\
& \times\left[\begin{array}{cc}
\mathbf{G}_{\mathrm{J}, 11}+\mathbf{G}_{\mathrm{J}, 12} & \mathbf{G}_{\mathrm{J}, 11} \cdot g_{\mathrm{C}}+\mathbf{G}_{\mathrm{J}, 12} \cdot g_{\mathrm{D}} \\
\mathbf{G}_{\mathrm{J}, 22} & \mathbf{G}_{\mathrm{J}, 22} \cdot g_{\mathrm{D}}
\end{array}\right] \\
& \times\left[\begin{array}{c}
\theta_{i+1} \\
\theta_{i+2}
\end{array}\right]
\end{aligned}
$$


or

$$
\begin{aligned}
& {\left[\begin{array}{l}
q_{j_{1}, i-1} \\
q_{j_{2}, i-1}
\end{array}\right]} \\
& =\left[\begin{array}{ccc}
g_{\mathrm{A}} & g_{\mathrm{A}}\left(\mathbf{G}_{\mathrm{J}, 11}+\mathbf{G}_{\mathrm{J}, 12}\right) & g_{\mathrm{A}}\left(\mathbf{G}_{\left.\mathrm{J}, 11 g_{\mathrm{C}}+\mathbf{G}_{\mathrm{J}, 12} g_{\mathrm{D}}\right)}\right. \\
g_{\mathrm{B}} & g_{\mathrm{B}} \mathbf{G}_{\mathrm{J}, 22} & g_{\mathrm{B}} \mathbf{G}_{\mathrm{J}, 22} g_{\mathrm{D}}
\end{array}\right] \\
& +\left[\begin{array}{c}
\theta_{1} \\
\theta_{i+1} \\
\theta_{i+2}
\end{array}\right]
\end{aligned}
$$

where the term $\left(G_{J, 11}+G_{J, 12}\right)$ is called the upper forward gain of jointed unit $\mathrm{J}$.

\subsection{Example}

For the GRM shown in Figure 1d, it can be seen that IU-1 precedes jointed unit JU-1 while TU-1 succeeds JU-1 in its upper sub-MTL. From Table I, it can be found that $g_{\mathrm{TU}-1}=e_{38}$. From Eq. (21), the angular displacements of links 8 and $2^{\prime}$ with respect to primary link 1 can be related to those of links 3 and 2 by substituting associated forward gains into Eq. (21) as

$$
\begin{aligned}
{\left[\begin{array}{l}
q_{8,1} \\
q_{2^{\prime}, 1}
\end{array}\right] } & =\left[\begin{array}{l}
1 \\
0
\end{array}\right] \theta_{2}+\left[\begin{array}{cc}
e_{38} & 0 \\
0 & 1
\end{array}\right]\left[\begin{array}{l}
q_{3,2} \\
q_{2,1}
\end{array}\right] \\
& =\left[\begin{array}{l}
1 \\
0
\end{array}\right] \theta_{2}+\left[\begin{array}{cc}
e_{38} & 0 \\
0 & 1
\end{array}\right]\left[\begin{array}{l}
\theta_{3} \\
\theta_{2}
\end{array}\right]
\end{aligned}
$$

The angular displacements of links 8 and $2^{\prime}$ with respect to primary link 1 of JU-1 can be related to those of links 5 and 4 by the forward gain matrix in Table II as

$$
\left[\begin{array}{l}
q_{5,1} \\
q_{4,1}
\end{array}\right]=\left[\begin{array}{cc}
e_{65} e_{76} e_{87} & \left(1-e_{65} e_{76}\right) \\
0 & e_{24}
\end{array}\right]\left[\begin{array}{l}
q_{8,1} \\
q_{2^{\prime}, 1}
\end{array}\right]
$$

From Eq. (17), angular displacements of the local inputs of the JU-1, links 5 and 4, with respect to primary link 1 can be related to those of the preconnecting links of the preceding IU-1, links 5 and 4 , with respect to primary link 0 as

$$
\left[\begin{array}{l}
q_{5,0} \\
q_{4,0}
\end{array}\right]=\left[\begin{array}{l}
1 \\
1
\end{array}\right] \theta_{1}+\left[\begin{array}{ll}
1 & 0 \\
0 & 1
\end{array}\right]\left[\begin{array}{l}
q_{5,1} \\
q_{4,1}
\end{array}\right]
$$

Substituting Eqs. (28) and (29) into Eq. (30) yields

$$
\begin{aligned}
{\left[\begin{array}{l}
q_{5,0} \\
q_{4,0}
\end{array}\right]=} & {\left[\begin{array}{ccc}
1 & e_{65} e_{76} e_{87}+e_{24}\left(1-e_{65} e_{76}\right) & e_{65} e_{76} e_{87} e_{38} \\
1 & e_{24} & 0
\end{array}\right] } \\
& \times\left[\begin{array}{c}
\theta_{1} \\
\theta_{2} \\
\theta_{3}
\end{array}\right]
\end{aligned}
$$

For the direct-drive disjointed MTL shown in Figure 1b, we have

$$
q_{1,0}=g_{\mathrm{IU}-1} \theta_{1}=\theta_{1}
$$

Hence, from Eqs. (31) and (32), angular displacement vectors associated with the actuator space and the joint space of the GRM with a jointed unit can be obtained as

$$
\begin{aligned}
{\left[\begin{array}{l}
q_{1,0} \\
q_{5,0} \\
q_{4,0}
\end{array}\right] } & =\left[\begin{array}{ccc}
1 & 1 & 1 \\
0 & e_{65} e_{76} e_{87}+e_{24}\left(1-e_{65} e_{76}\right) & e_{24} \\
0 & e_{65} e_{76} e_{87} e_{38} & 0
\end{array}\right]^{T}\left[\begin{array}{l}
\theta_{1} \\
\theta_{2} \\
\theta_{3}
\end{array}\right] \\
& =\mathbf{A}^{T}\left[\begin{array}{l}
\theta_{1} \\
\theta_{2} \\
\theta_{3}
\end{array}\right]
\end{aligned}
$$

where $\mathbf{A}^{T}$ represents the transpose of the structure matrix of the GRM. ${ }^{3}$

\section{THE JUMP-OVER EFFECT}

The equation relating the joint torques to the actuator torques ${ }^{3}$ is

$$
\tau=\mathbf{A} \xi
$$

where $\tau$ and $\xi$ are torque vectors at joint space and actuator space, respectively.

From Eqs. (27) and (34), torque relations of a jointed unit $\mathrm{J}$ with preceding disjointed units $\mathrm{A}$ and $\mathrm{B}$ and succeeding disjointed units $\mathrm{C}$ and $\mathrm{D}$ can be obtained as

$$
\begin{aligned}
{\left[\begin{array}{c}
\tau_{i, i-1} \\
\tau_{i+1, i} \\
\tau_{i+2, i+1}
\end{array}\right]=} & {\left[\begin{array}{cc}
g_{\mathrm{A}} & g_{\mathrm{B}} \\
g_{\mathrm{A}}\left(\mathbf{G}_{\mathrm{J}, 11}+\mathbf{G}_{\mathrm{J}, 12}\right) & g_{\mathrm{B}} \mathbf{G}_{\mathrm{J}, 22} \\
g_{\mathrm{A}}\left(\mathbf{G}_{\mathrm{J}, 11} g_{\mathrm{C}}+\mathbf{G}_{\mathrm{J}, 12} g_{\mathrm{D}}\right) & g_{\mathrm{B}} \mathbf{G}_{\mathrm{J}, 22} g_{\mathrm{D}}
\end{array}\right] } \\
& \times\left[\begin{array}{c}
\xi_{j_{1}, i-1} \\
\xi_{j_{2}, i-1}
\end{array}\right]
\end{aligned}
$$


Chang and Tsai $^{3}$ claimed that nonzero elements in each column of a structure matrix must be consecutive, i.e., the influences of an actuator to various joints along its associated MTL must be consecutive. However, from Eq. (35), it can be seen that it is possible to have an intermediate zero element in a column of the coefficient matrix by letting $g_{\mathrm{A}}\left(\mathbf{G}_{\mathrm{J}, 11}\right.$ $+\mathbf{G}_{\mathrm{J}, 12}$ ) equal zero. Hence, it is possible for an actuator to drive a remote joint without the influence on an intermediate joint in the same MTL, and the phenomenon is referred to as the jump-over effect. Since the forward gain of disjointed unit A, $g_{\mathrm{A}}$, has a nonzero value, the condition to make $g_{\mathrm{A}}\left(\mathbf{G}_{\mathrm{J}, 11}+\mathbf{G}_{\mathrm{J}, 12}\right)$ equal to zero is the same as that to make the upper forward gain $\left(G_{\mathrm{J}, 11}+\mathbf{G}_{\mathrm{J}, 12}\right)$ equal to zero. From Table II, it can be found that only the upper forward gains of JU-1 and JU-3 contain multiple terms and thus are capable of being set to zero by proper gear train design.

Suppose that a 3-DOF GRM with a jointed unit is to be designed based on the structure matrix in the left-hand side of Figure 3a. It is desired to have an intermediate zero element in the second MTL as shown to the right of Figure 3a. To achieve the jump-over effect, the jointed unit JU-3 is chosen as the kernel of a set of jointed MTLs. In Figure 3a, the columns corresponding to the associated jointed MTLs are shaded and the locations of the upper and lower forward gains in the structure matrix are marked by a dashed rectangle.

In Figure 3a there is a nonzero element succeeding the upper forward gain in the structure matrix. Hence, a succeeding disjointed unit is required to connect JU-3 in the upper sub-MTL. On the other hand, since there is no nonzero element succeeding the lower forward gain, no succeeding disjointed unit is required to connect JU-3 in the lower subMTL. Besides, since nonzero elements precede both the upper and lower forward gains in the structure matrix, preceding disjointed units are required to connect JU-3 in both sub-MTLs. Transmission unit TU-1 and input unit IU-1 are chosen as the succeeding and preceding disjointed units from Table $\mathrm{Ib}$ and $\mathrm{Ia}$, respectively. Figure $3 \mathrm{~b}$ shows the arrangement of jointed and disjointed units within the jointed MTLs.

It can be seen that the column representing the disjointed MTL in the structure matrix has only one nonzero element at the first joint. Thus, a one-joint two-link disjointed unit IU-1 is chosen as the direct-drive type disjointed MTL. By adding the disjointed MTL to the jointed MTLs followed by

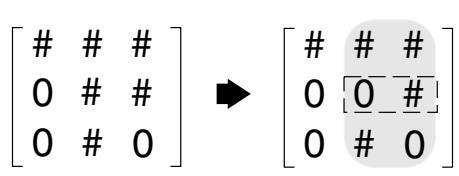

(a)

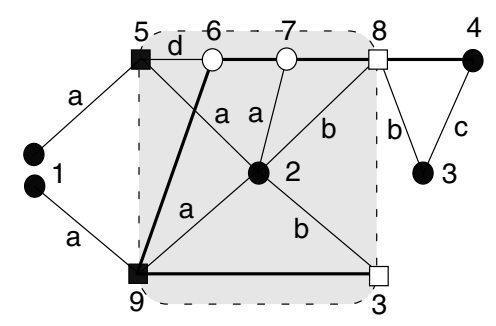

(b)

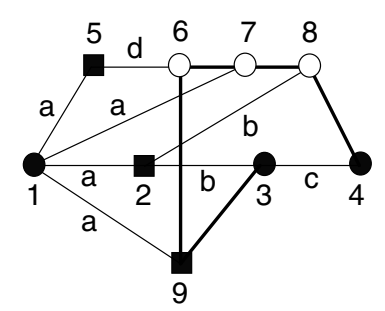

(c)

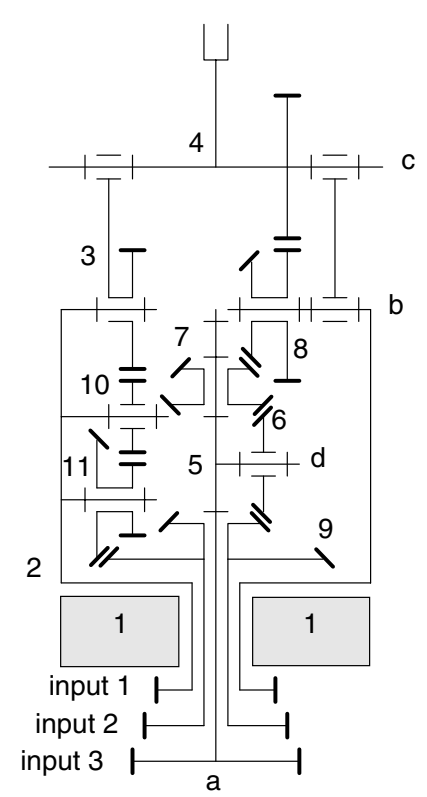

(d)

Figure 3. A 3-DOF GRM with a jointed unit. (a) The structure matrix. (b) The arrangement sequences of the units. (c) The canonical graph representation. (d) The functional representation. 
rearranging coaxial links, the canonical graph representation of this 3-DOF GRM with a jointed unit can be obtained and is shown in Figure 3c. From Eqs. (32) and (35), the structure matrix of the resultant GRM with a jointed unit can be written as

$$
\mathbf{A}=\left[\begin{array}{ccc}
g_{\mathrm{IU}-1} & g_{\mathrm{IU}-1} & g_{\mathrm{IU}-1} \\
0 & g_{\mathrm{IU}-1}\left(\mathbf{G}_{\mathrm{JU}-3,11}+\mathbf{G}_{\mathrm{JU}-3,12}\right) & \mathbf{G}_{\mathrm{JU}-3,22} \\
0 & g_{\mathrm{IU}-1} \mathbf{G}_{\mathrm{JU}-3,11} g_{\mathrm{TU}-1} & 0
\end{array}\right]
$$

In Eq. (36), the forward gains of associated disjointed units and the forward gain matrix of JU-3 can be found in Tables I and II, respectively. To eliminate the compound element in Eq. (36), the following equation must be satisfied:

$$
\mathbf{G}_{\mathrm{JU}-3,11}+\mathbf{G}_{\mathrm{JU}-3,12}=\frac{e_{87}}{1-e_{67} e_{96}}-\frac{e_{96} e_{67} e_{39}}{1-e_{67} e_{96}}=0
$$

Equation (37) leads to the following two conditions:

$$
\begin{gathered}
e_{67} e_{96} \neq 1 \\
e_{87}=e_{96} e_{67} e_{39}
\end{gathered}
$$

Thus, joint torques and actuator torques of the GRM with a jointed unit can be related as

$$
\left[\begin{array}{l}
\tau_{2,1} \\
\tau_{3,2} \\
\tau_{4,3}
\end{array}\right]=\left[\begin{array}{ccc}
1 & 1 & 1 \\
0 & 0 & e_{39} \\
0 & \frac{e_{87} e_{58}}{1-e_{67} e_{96}} & 0
\end{array}\right]\left[\begin{array}{l}
\xi_{2,1} \\
\xi_{5,1} \\
\xi_{9,1}
\end{array}\right]
$$

For the GRM with a jointed unit shown in Figure 3d, Eq. (40) shows that the second MTL transmits input torque to both the first and the third joints while the second joint is jumped over without any influence caused by the actuator on link 5 . Figure $3 \mathrm{~d}$ shows a functional representation of the GRM with a jointed unit with link 1 as the ground link. It can be seen that link 6 is designed as a bevel gear to mesh with bevel gears 7 and 9 simultaneously. Thus, gear ratios $e_{67}$ and $e_{96}$ are of opposite signs and Eq. (38) is always satisfied. Note that in Figure $3 d$, gear links 10 and 11 are added as idlers to extend the center distance between links 9 and
3 and to ensure the sign requirement as stated in Eq. (39).

\section{CONCLUSION}

In this article, a systematic methodology for the kinematic analysis of GRMs with a jointed unit is developed. The kinematic structure of a GRM with a jointed unit can be decomposed into an equivalent open-loop chain and jointed and disjointed MTLs. A set of jointed MTLs can be further decomposed into a jointed unit and several disjointed units while a disjointed MTL consists of disjointed units only. Relations between local input link(s) and output link(s) of each jointed unit are formulated to derive the kinematic propagation properties between connected units. Accordingly, input angular displacements of a GRM with a jointed unit can be systematically represented in terms of joint angular displacements by a unit-by-unit evaluation procedure without the need to solve a set of linear equations simultaneously. It is shown that the jump-over effect can be achieved such that the influence of an actuator to an articulated joint can be eliminated without terminating the transmission to its succeeding joint by proper joint unit selection and gear train design.

\section{REFERENCES}

1. C.C. Lin and L.W. Tsai, The development of an atlas of bevel-gear-type spherical wrist mechanisms, Proc First National Conf Applied Mechanisms and Robotics, Cincinnati, OH, 1989, Paper No. AMR-2A-3.

2. N.P. Belfiore and L.W. Tsai, A new methodology for structural synthesis of geared robotic wrists, Proc Second National Conf Applied Mechanisms and Robotics, Cincinnati, OH, 1991, Paper No. VIB 5.

3. S.L. Chang and L.W. Tsai, Topological synthesis of articulated gear mechanisms, IEEE Trans Robotics Automat 6 (1990), 97-103.

4. D.Z. Chen and S.C. Shiue, Topological synthesis of geared robotic mechanism, ASME J Mech Design 120 (1998), 230-239.

5. D.Z. Chen, Y.C. Huang, and D.W. Du, Geared robot manipulators with a jointed unit: topological synthesis and its aplication, Int J Robotic Res 19 (2000), 183-194.

6. F. Freudenstein, R.W. Longman, and C.K. Chen, Kinematic analysis of robotic bevel-gear trains, ASME 
Trans J Mechanisms, Transmissions Automat Design 106 (1984), 371-375.

7. F.L. Litvin and Z. Yi, Robotic bevel gear train, Int J Robotics Res 5 (1986), 75-81.

8. R. Ma and K.C. Gupta, On the motion of oblique bevel geared robot wrists, J Robotic Syst 6 (1989), 509-520.

9. L.W. Tsai, The kinematics of spatial robotic bevel-gear trains, IEEE Trans Robotics Automat 4 (1988), 150-156.

10. D.Z. Chen, Kinematic analysis of geared robot manip- ulators by the concept of structural decomposition, Mechanism Machine Theory 33 (1998), 975-986.

11. L.W. Tsai and C.C. Lin, The creation of non-fractionated two-degree-of-freedom epicyclic gear trains, ASME J Mechanisms Transmissions Automat Design 111 (1989), 524-529.

12. F. Freudenstein, Kinematics and statics of a coupled epicyclic spur-gear train, Mechanism Machine Theory 7 (1972), 263-275. 